\title{
Speech Enhancement using an Adaptive Gain Equalizer with Frequency Dependent Parameter Settings
}

\author{
Nils Westerlund \\ Mattias Dahl \\ Nils.Westerlund@bth.se \\ Mattias.Dahl@bth.se \\ Ingvar Claesson \\ Ingvar.Claesson@bth.se \\ Blekinge Institute of Technology \\ School of Engineering \\ Applied Signal Processing Group \\ Ronneby, Sweden
}

February, 2004

\begin{abstract}
This paper presents an enhanced noise reduction method for speech communication where the input signal is divided into a number of subbands that are individually weighted in time domain according to the short time Signal-to-Noise Ratio estimate (SNR) in each subband at every time instant. Instead of focusing on suppression the noise, the method is focusing on speech enhancement.

The subband based method is extended with frequency dependent parameter settings, equipping the user with further tweaking possibilities. Hence, making the algorithm even more versatile and applicable in different noise situations.

The method has proven to be advantageous since it offers low complexity, low delay and low distortion. Also, there is no need for a Voice Activity Detector (VAD). The method is stand-alone and works regardless of speech coding schemes and other surrounding adaptive systems.
\end{abstract}

\section{Introduction}

Personal communication of today is impaired by nearly ubiquitous noise. Speech communication becomes difficult under these conditions; speech sounds are masked by the noise and speech features such as overall quality and intelligibility may be degraded. Today, a great deal of our personal communication is performed using some sort of communication equipment, such as cellular phones 
and inter-com devices. With the prosperity of such portable communication devices, speech enhancement has received an increased attention. Noise corrupted speech does indeed force the user of the communication equipment to strain both hearing and voice. Altogether, acoustic noise directly affects human communication, and also dramatically decreases the performance of speech coding and speech recognition algorithms. This urges for effective speech enhancement methods.

Speech enhancement refers to the restoration of clean speech. The main objective of speech enhancement is to improve one or more perceptual aspects of speech, such as human or machine speech recognition or degree of listener fatigue.

An early and fundamental method for noise reduction, was to use the theory of the optimum Wiener filter [1]. Given a desired signal and an input signal, the Wiener filter produces the minimum mean square error estimate of the desired signal. The Wiener filter can also be adapted to a non-stationary noise environment: Adaptive algorithms such as the Least Mean Square (LMS) and Recursive Least Squares (RLS) are well known examples and also widely used [2], [3].

Today, a frequently used digital method for effective noise reduction in speech communication, is spectral subtraction [4], [5]. This frequency domain method is based on the Fast Fourier Transform and is a non-linear, yet straightforward way of reducing unwanted broadband noise acoustically added to a signal. The noise bias is estimated in frequency domain during speech pauses and then subtracted from the noisy speech spectra. The quality of the noise bias estimate is crucial for the final result and a VAD is needed to detect non-speech activity. Properly tweaked spectral subtraction is a powerful tool for noise reduction in acoustic signals and a number of enhancements of the original algorithm have been proposed during the years to reduce delay and so-called musical tones [6], [7], [8]. Efforts have also been made to reduce or eliminate the need for VADs $[9],[10]$.

Multi microphone techniques such as microphone arrays, have also been investigated in order to suppress a disturbance both spatially and temporally by means of adaptive beamforming and signal separation [11], [12], [13].

A general, stand-alone, time-domain method for speech enhancement is described in [14],[15]. In this method, the input signal is presented to a SingleInput-Single-Output (SISO) system in which the signal is divided into a number of subbands. These subbands are then individually and temporally weighted according to an estimate of the SNR in each subband at every time instant. The weighting of the subbands is analogue to adaptively increase the gain of a graphical audio equalizer in those subbands where speech is dominant. Hence the name Adaptive Gain Equalizer (AGE). The AGE acts as a speech booster, only active when speech is present and it remains idle when only noise is present. The method has proven to be robust, flexible and versatile and results in speech enhancement with low speech distortion without the requirement of a VAD.

In this paper an enhanced version of the AGE is presented, in which the parameters are made fully frequency dependent, equipping the user of the method with extended tweaking possibilities in order to make the method even more versatile and applicable to various noise situations. 


\section{Problem Formulation and Method}

The basic idea behind the proposed method, is that a speech signal corrupted by uncorrelated, additive noise can be divided into a number of subbands and that each of these subbands can be individually and adaptively boosted according to an SNR estimate in that particular subband signal.

A short term average for speech tracking and a long term average for background noise floor level tracking are calculated simultaneously. Using the quotient of these quantities, a gain function is achieved that weights the subband signal directly according to a subband signal SNR estimate at that particular time instant. If only noise is present in the signal, the noise floor level estimate and the short term average will be approximately equal. Hence the quotient of these two measures will be unity and no alteration of the subband signal will be performed. If speech is present, the short term average will increase but the noise floor level estimate will remain approximately unchanged. Hence, the quotient will become larger than unity, amplifying the signal in the subband at hand.

The proposed method acts as a speech booster that is adaptively looking for subband speech energy to boost. Speech energy manifests itself as highly non-stationary excursions in input level. If there are no such excursions, no speech boosting will take place; the AGE will remain idle. This in turn results in distortion free background noise when there is no speech activity and, thanks to masking effects, inaudible background distortion during speech activity. Artifacts such as musical tones, a common problem when using spectral subtraction, are more or less eliminated.

Another major advantage is the fact that since the method is based upon a continuous estimate of the SNR in each subband, a VAD renders superfluous. Traditional VADs are difficult to tune and, if applied to low SNR speech, often result in artifacts. Hence, it is desirable to eliminate this step in the traditional speech enhancement chain. In the proposed method, on the contrary it is possible that a low SNR subband signal may indeed contribute with some speech information during speech activity.

Altogether, the advantages are manyfold when using this method:

- Increased speech quality - The resulting output signal is natural sounding and with a minimum of distortion and artifacts.

- No VAD is required - The speech enhancement is performed continuously in each subband.

- Flexible and versatile - Can be implemented either on digital or analog circuits.

- Robust - Requires a minimum of tweaking for good performance in various noise environments.

- Stand-Alone - The method works independent of different speech coding schemes or other adaptive algorithms.

- Low complexity - Few MIPS. 


\subsection{The Proposed Method}

Suppose we have an acoustical discrete time speech signal denoted $s(n)$ and a discrete time noise signal denoted $w(n)$. The noise corrupted speech signal $x(n)$ can then be written as

$$
x(n)=s(n)+w(n)
$$

By filtering the input signal $x(n)$ using a bank of $K$ bandpass filters, $h_{k}(n)$, the signal is divided into $K$ subbands, each denoted by $x_{k}(n)$ where $k$ is the subband index. This filtering operation can be written in time domain as

$$
x_{k}(n)=x(n) * h_{k}(n)
$$

where $*$ is the convolution operator. In the ideal case, the original signal can then be described as

$$
x(n)=\sum_{k=0}^{K-1} x_{k}(n)=\sum_{k=0}^{K-1} s_{k}(n)+w_{k}(n)
$$

i.e. $x_{k}(n)=s_{k}(n)+w_{k}(n)$ where $s_{k}(n)$ is the speech part subband $k$ and $w_{k}(n)$ is the noise part subband $k$. The output $y(n)$ is formed by

$$
y(n)=\sum_{k=0}^{K-1} G_{k}(n) x_{k}(n)
$$

where $G_{k}(n)$ is a weighting function that amplifies the band $k$ during speech activity. Since $G_{k}(n)$ introduces a gain to each subband, the function will be denoted gain function for the remainder of this paper.

Our desire is now to find a gain function that weights the input signal subbands using the ratio between $s_{k}(n)$ and $w_{k}(n)$, i.e. a short term SNR estimate. A block scheme illustrating the subband decomposition, weighting and final summation is shown in Fig. 1.

The gain function in each subband is found by using a ratio of a short term exponential magnitude average, $A_{x, k}(n)$ based on $\left|x_{k}(n)\right|$, and an estimate of the noise floor level, $\underline{A}_{x, k}(n)$. The short term average in subband $k, A_{x, k}(n)$, is calculated as

$$
A_{x, k}(n)=\left(1-\alpha_{k}\right) A_{x, k}(n-1)+\alpha_{k}\left|x_{k}(n)\right|
$$

where $\alpha_{k}$ is a small positive constant controlling how sensitive the algorithm should be to rapid changes in input signal amplitude in subband $k$, i.e. a smoothing factor. Human speech can be considered approximately short time stationary [5], [16], and the constant $\alpha_{k}$ should be chosen with this in mind. A suitable value for $\alpha_{k}$ can be estimated using the following equation:

$$
\alpha_{k}=\frac{1}{T_{s, k} F_{s}}
$$

where $F_{s}$ is the sampling frequency and $T_{s, k}$ is a time constant.

The slowly varying noise floor level estimate for each subband $k, \underline{A}_{x, k}(n)$, is calculated according to

$$
\begin{aligned}
& \underline{A}_{x, k}(n)= \\
& =\left\{\begin{array}{rrr}
\left(1+\beta_{k}\right) \underline{A}_{x, k}(n-1) & \text { if } & A_{x, k}(n)>\underline{A}_{x, k}(n-1) \\
A_{x, k}(n) & \text { if } & A_{x, k}(n)
\end{array}\right.
\end{aligned}
$$




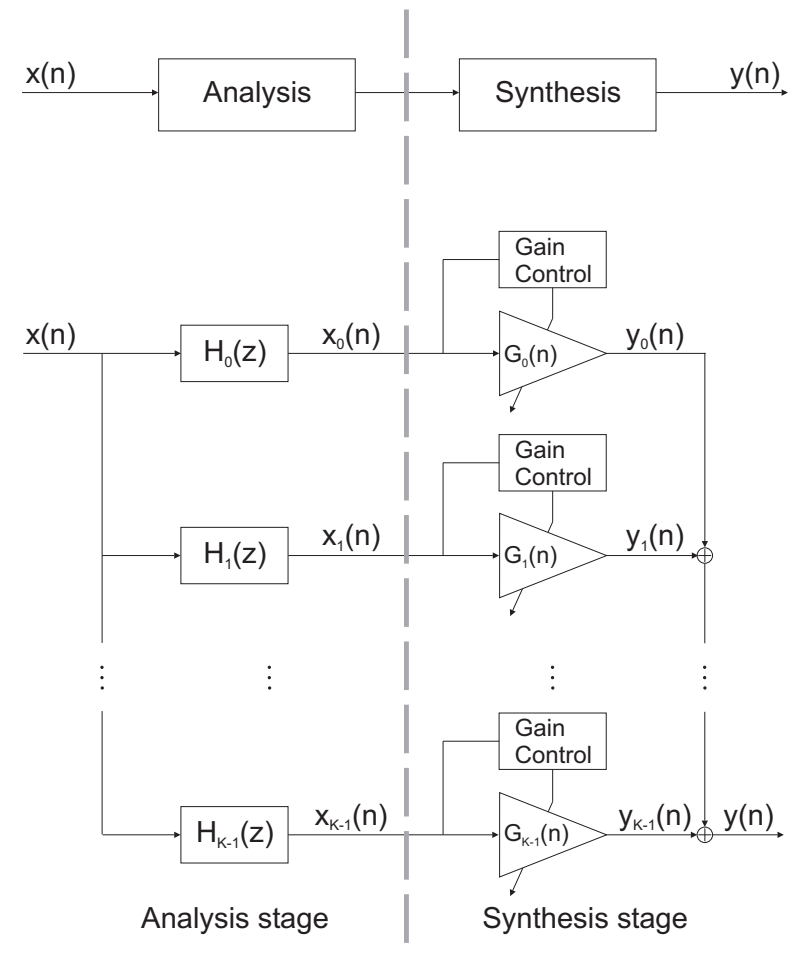

Figure 1: Signal decomposition-weighting-assembly.

where $\beta_{k}$ is a small positive constant controlling how fast the noise floor level estimate in subband $k$ will adapt to changes in the noise environment. Note that $A_{x, k}(n) \geqslant \underline{A}_{x, k}(n)$ and that $\beta_{k} \ll \alpha_{k}$.

The variables $A_{x, k}(n)$ and $\underline{A}_{x, k}(n)$ are used to form the gain function $G_{k}(n)$ according to

$$
G_{k}(n)=\left(\frac{A_{x, k}(n)}{\underline{A}_{x, k}(n)}\right)^{p_{k}}, \quad p_{k} \geqslant 0, \underline{A}_{x, k}(n)>0
$$

where $p_{k}$ decides the gain raise individually applied to each of the subband signals. The resulting speech enhanced output signal $y(n)$ is then calculated as in (4).

Since the calculation of $G_{k}(n)$ involves division, care must be taken to ensure that the quotient does not become excessively large due to a small $\underline{A}_{x, k}(n)$. In a situation with a fair SNR, $G_{k}(n)$ will become considerable if no limit is imposed on this function, resulting in unacceptable high speech amplification. A limiter can be imposed on $G_{k}(n)$ as follows:

$$
G_{k}(n)=\left\{\begin{array}{lll}
G_{k}(n) & \text { if } & G_{k}(n) \leqslant L_{k} \\
L_{k} & \text { if } \quad G_{k}(n)>L_{k}
\end{array}\right.
$$

where $L_{k}$ is some positive constant, e.g. expressed in decibels. 


\section{Evaluation}

A general mathematical description of the method was given in section 2.1 where a number of subband dependent parameters were introduced. These parameters are now set to subband-dependent values for evaluation purposes. Since the algorithm offers four controlling parameters $-T_{s, k}, \beta_{k}, L_{k}$ and $p_{k}$ - the degrees of freedom regarding parameter combinations rapidly becomes large. Hence, the approach in this paper was to set all parameters but the one under investigation to a suitable default value. These default values are listed in Tab. 1.

All experimental evaluations have been performed on phonetically balanced sentences recorded on site in a moving car at highway speed. The sampling frequency was set to $8 \mathrm{kHz}$.

\subsection{The Filter Bank}

In section 2.1, a bank of bandpass filters was used to divide the input signal into $k$ subbands. Intentionally, this was a quite general description. The linearly spaced FIR filters used in this paper are designed with the window method using Hamming windows. This method results in causal, symmetric impulse responses with linear phase. In this paper, a filter bank consisting of ten such subbands is used.

\subsection{The Short Term Average}

In order to effectively calculate a short term average magnitude of the input signal, a single-pole recursion was used as described in section 2.1, (5). The parameter $T_{s, k}$ was made subband dependent and three settings of $T_{s, k}$ were evaluated. These are listed in Tab. 1.

The parameter $T_{s, k}$ controls how sensitive the short term average $A_{x, k}$ should be to incoming speech. I.e. the parameter controls the "attack" and "decay" of the short term average.

\subsection{The Noise Floor Level Estimate}

The noise floor level estimate described in section 2.1, (8), is designed to adaptively track the background noise level. The value of $\underline{A}_{x, k}(n)$ is dependent on the short term average, $A_{x, k}(n)$. The positive constant $\beta_{k}$ controls how fast the noise floor level estimate will adapt to changes in the noise environment. For evaluation purposes, this constant $\beta_{k}$ was made subband dependent in the same manner as the short term average control parameter $T_{s, k}$. The settings are listed in Tab. 1.

\subsection{The Gain Function}

As described in section 2.1, (8), the gain function $G_{k}(n)$ is the ratio of the short term average and the noise floor level estimate. Since we are dealing with a ratio, care must be taken to avoid singularities. In a communication environment with very little background noise, the SNR in in some (or all) subbands would become excessively large, resulting in an unacceptable high speech amplification. The 


\begin{tabular}{|c||c|c|c|}
\hline \multicolumn{1}{|c||}{} & \multicolumn{3}{c|}{ Value } \\
\hline Parameter & Case I (Default) & Case II & Case III \\
\hline$\left[T_{s, 1} \ldots T_{s, 10}\right]$ & {$[0.03 \ldots 0.03]$} & {$[0.01 \ldots 0.1]$} & {$[0.1 \ldots 0.01]$} \\
\hline$\left[\beta_{1} \ldots \beta_{10}\right]$ & {$\left[10^{-6} \ldots 10^{-6}\right]$} & {$\left[10^{-7} \ldots 10^{-5}\right]$} & {$\left[10^{-5} \ldots 10^{-7}\right]$} \\
\hline$\left[L_{1} \ldots L_{10}\right]$ & {$\left[10^{\frac{10}{20}} \ldots 10^{\frac{10}{20}}\right]$} & {$\left[10^{\frac{5}{20}} \ldots 10^{\frac{20}{20}}\right]$} & {$\left[10^{\frac{20}{20}} \ldots 10^{\frac{5}{20}}\right]$} \\
\hline$\left[p_{1} \ldots p_{10}\right]$ & {$[1 \ldots 1]$} & {$[0.5 \ldots 2]$} & {$[2 \ldots 0.5]$} \\
\hline
\end{tabular}

Table 1: Subband dependent settings.

remedy to this problem is to use an upper limit imposed on the gain function $G_{k}(n)$ as described in section 2.1, (9).

This gain limiter is set to one of the three cases listed in Tab. 1.

An exponential weighting of $G_{k}(n)$ is performed by raising the ratio $A_{x, k}(n) / \underline{A}_{x, k}(n)$ to a power of $p_{k}$, as in section $2.1,(8)$. The values of the exponential weighting are chosen in the same manner as the other parameters, and the settings are listed in Tab. 1.

\section{Results}

Experimental results including informal listening tests show that the choice of the constants $T_{s, k}$ and $\beta_{k}$ is crucial to the speech enhancement performance and the resulting speech quality.

A relatively small $\alpha_{k}$ results in unnatural sounding speech with remaining artifacts. A very large $\alpha_{k}$ results in a short term average that reacts too slowly to incoming signal amplitude variations. Hence, the speech attacks will be cropped and, in addition, the speech amplification in subbands with a small amount of noise will be limited.

The parameter $\beta_{k}$ also has a fundamental effect on the final speech enhancement result. A small $\beta_{k}$ results in a noise floor level estimate that reacts rapidly to changes in the noise environment. The disadvantage is that the noise floor also reacts to incoming speech. A larger $\beta_{k}$ results in a more stable noise floor estimate but also in slower convergence and poor noise level tracking capabilities in non-stationary environments.

Since there is probably less speech energy in higher subbands, it might be desirable to set beta $a_{k}$ to a smaller value in these regions.

\subsection{Speech Enhancement Performance}

As mentioned earlier, the method does not focus on noise suppression but rather on speech enhancement. It is important to notice the fact that we are actually not trying to improve the SNR by removing noise. Instead, the SNR is improved by amplifying the speech on a subband basis.

Experimental results shows that the number of subbands is not crucial for most types of noise. However, a more complex noise environment, e.g. narrow band noise or even periodic disturbances, may be more efficiently suppressed using a larger number of subbands.

Furthermore, the time constants that controls the short term average should be kept in the range of speech pseudo-stationarity time, i.e. about 20-30 ms [5]. 

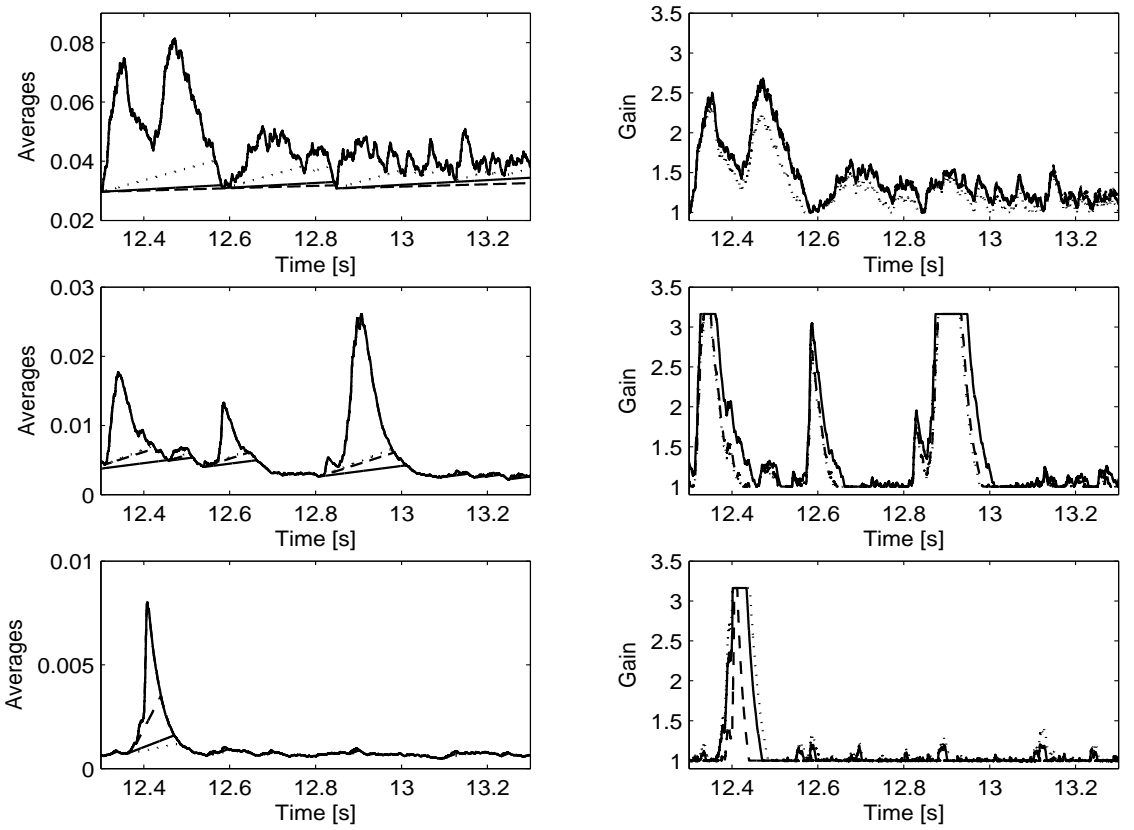

Figure 2: Varying $\beta_{k}$. (Solid line) Case I, uniform parameters. (Dashed line) Case II, increasing parameter value. (Dotted line) Case III, decreasing parameter value.
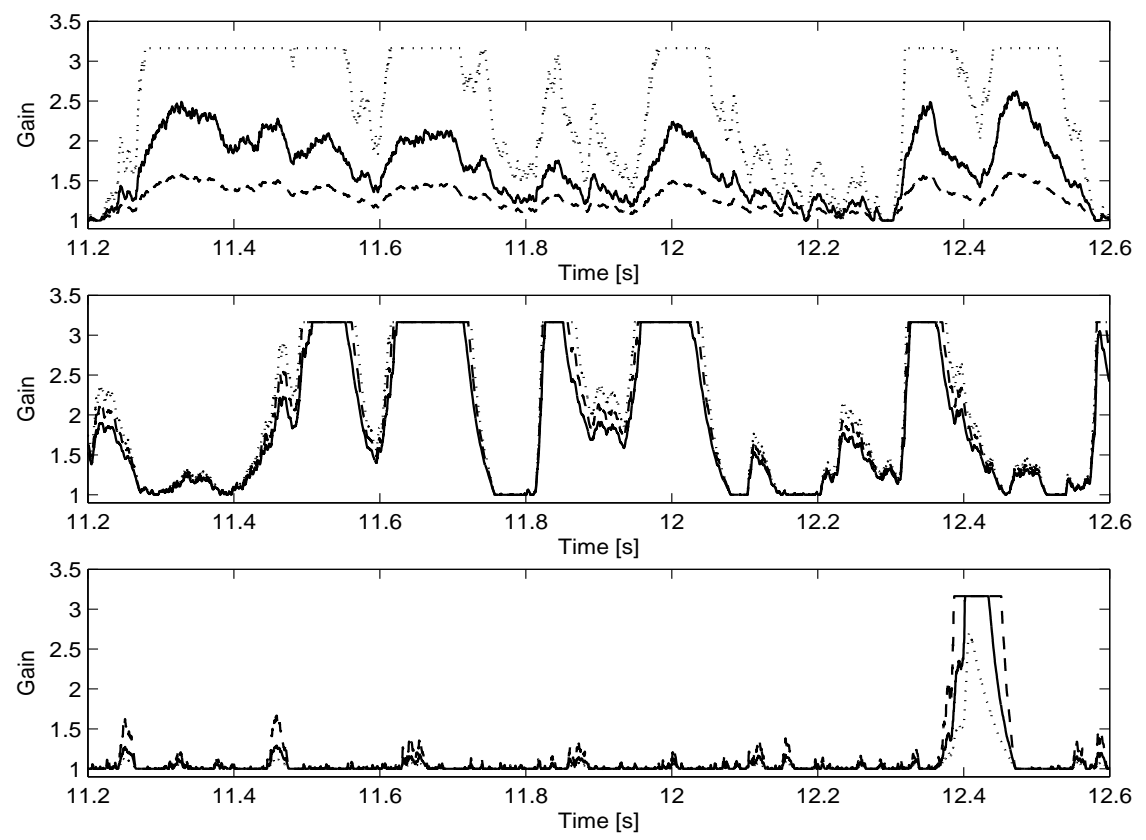

Figure 3: Varying $p_{k}$. (Solid line) Case I, uniform parameters. (Dashed line) Case II, increasing parameter value. (Dotted line) Case III, decreasing parameter value. 


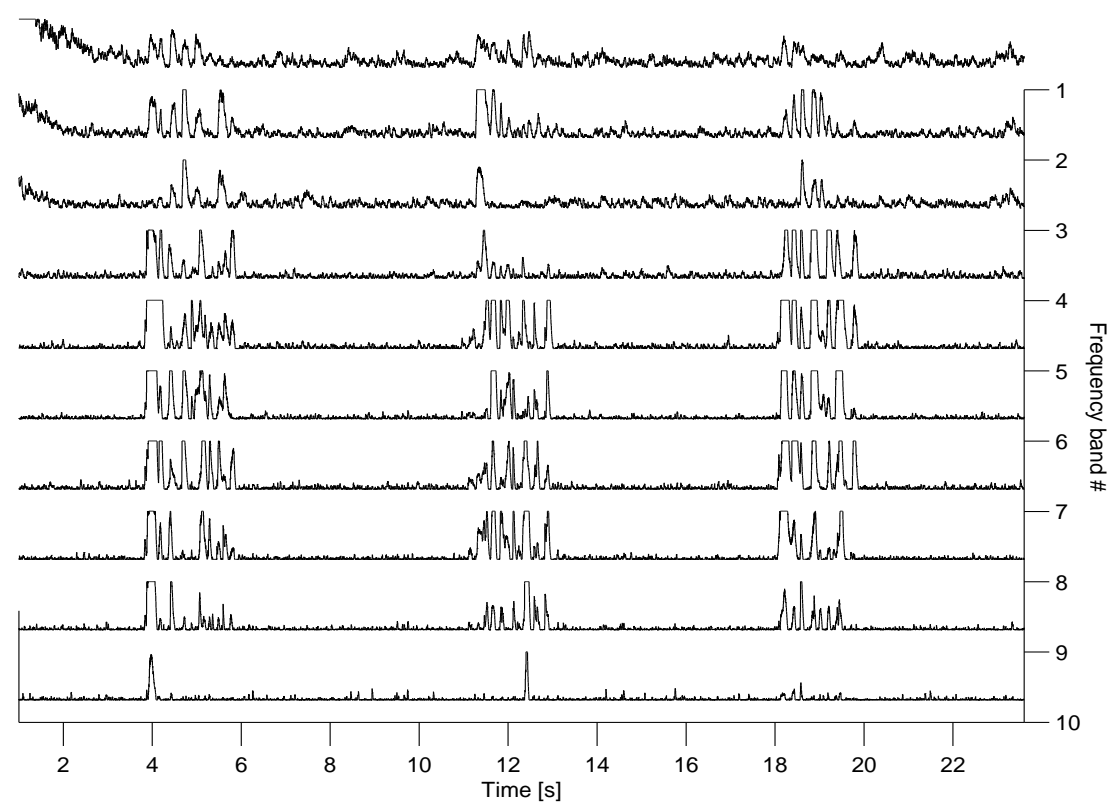

Figure 4: Varying $L_{k}$. Uniform parameter values, i.e. Case I.

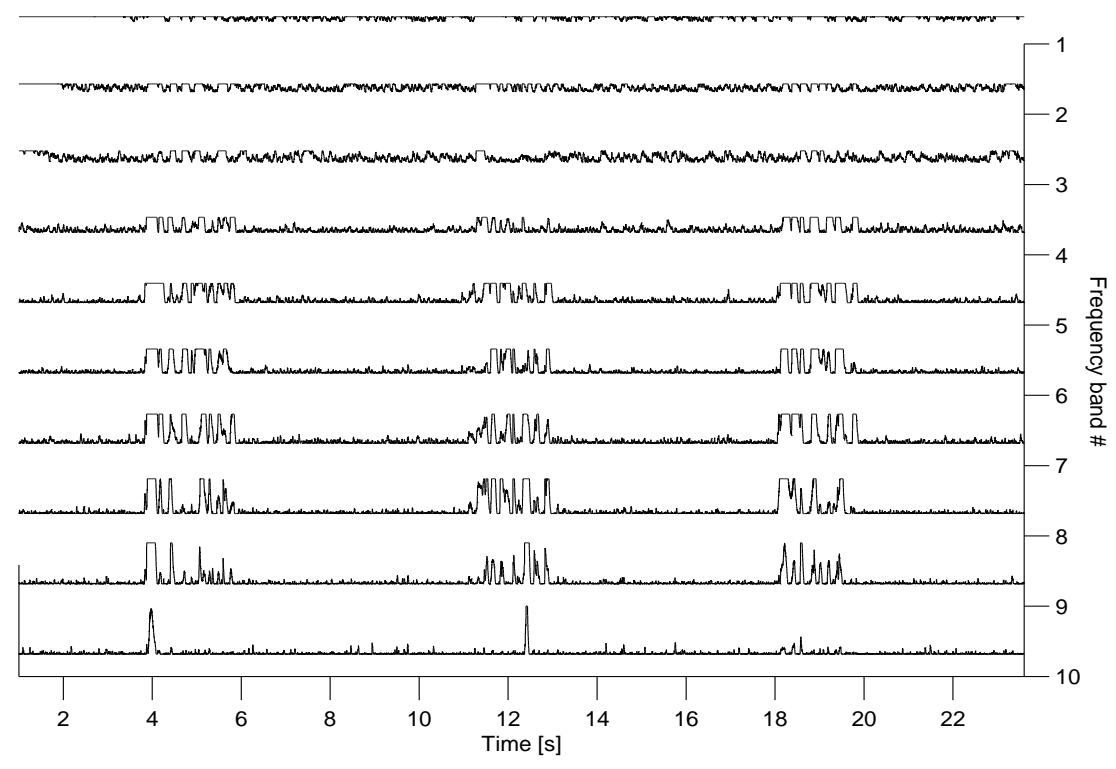

Figure 5: Varying $L_{k}$. Increasing parameter values, i.e. Case II. 


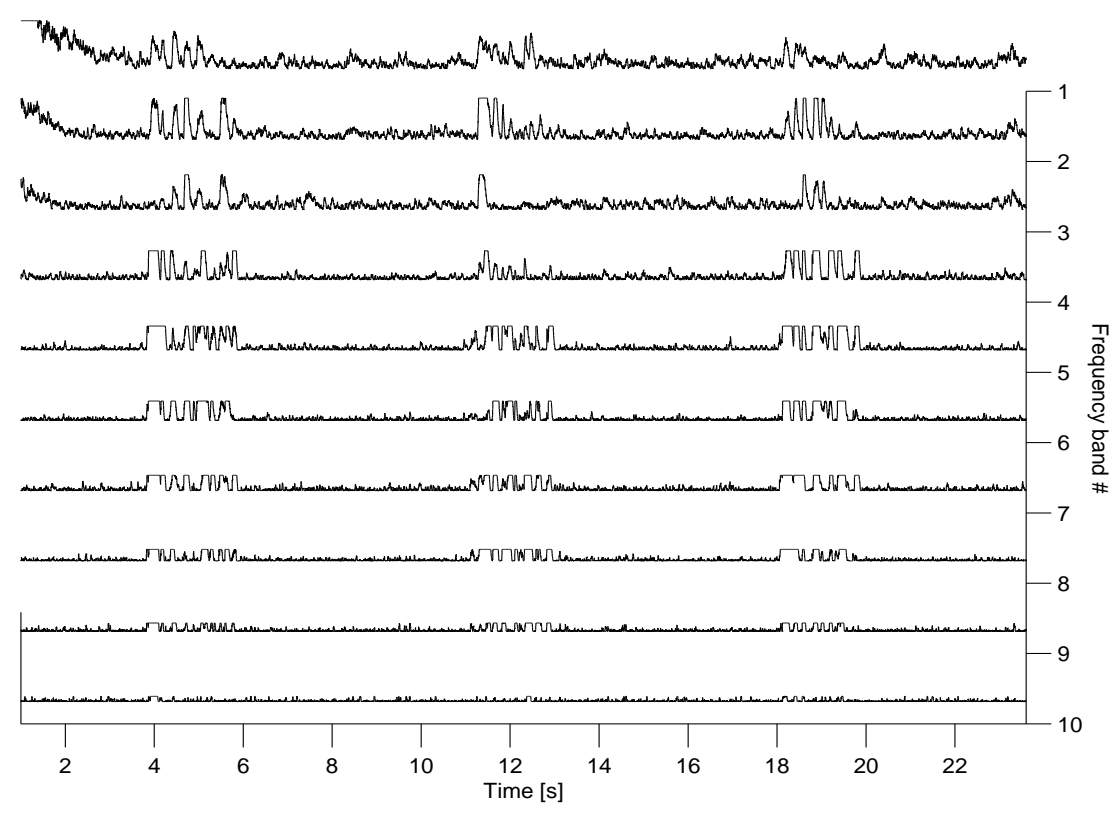

Figure 6: Varying $L_{k}$. Decreasing parameter value, i.e. Case III.

The noise floor level estimate controlling parameter, $\beta_{k}$, can be varied around $10^{-6}$ depending on the desired effects. The upper bounding of the gain function $G(n)$ affects the resulting speech distortion and should be kept within 5-20 dB. A larger amplification of the signal may result in a piercing sounding output speech.

\section{Conclusions}

A speech enhancement algorithm of today must be flexible yet robust, easily implemented yet computationally efficient and, in addition, it must be versatile and applicable to many different noise situations. The method described in this paper fulfills all of these criterions: Thanks to the straightforward basic underlying idea, the method is both flexible, robust and easily implemented. The filter bank structure used in this paper was an FIR filter bank but principally, any filter bank could be employed to obtain desired features and characteristics. In addition, experiments has shown that the method is easily configured and adapted to different noise situations with a minimum of tweaking.

Finally, the fact that the need for a VAD is eliminated, makes the method highly robust and a strong competitor (or complement) to existing noise reduction methods.

\section{References}

[1] M. H. Hayes, Statistical Digital Signal Processing and Modeling, Wiley, 1996. 
[2] B. Widrow and S. D. Stearns, Adaptive Signal Processing, Prentice-Hall, New Jersey, 1985.

[3] S. Haykin, Adaptive Filter Theory, Prentice-Hall, New Jersey, 1996.

[4] S. F. Boll, "Suppression of acoustic noise in speech using spectral subtraction," IEEE Trans. Acoust. Speech and Sig. Proc., vol. ASSP-27, pp. 113-120, April 1979.

[5] J. R. Deller Jr., J. G. Proakis, and J. H. L. Hansen, Discrete time processing of speech signals, Macmillan Publishing Company, 1993.

[6] Y. Ephraim and D. Malah, "Speech enhancement using a minimum mean-square error short-time spectral amplitude estimator," IEEE Trans. Acoust. Speech and Sig. Proc., vol. ASSP-32, pp. 1109-1121, December 1984.

[7] O. Cappé, "Elimination of the musical noise phenomenon with the ephraim and malah noise suppressor," IEEE Trans. Speech and Audio Proc., vol. 2, no. 2, pp. 345-349, 1994.

[8] H. Gustafsson, S. Nordholm, and I. Claesson, "Spectral subtraction using reduced delay convolution and adaptive averaging," IEEE Trans. Speech and Audio Proc., vol. 9, no. 8, 2001.

[9] R. Martin, "Spectral subtraction based on minimum statistics," in SIGNAL PROCESSING VII: Theories and Applications, P. Grant W. Sandham M. Holt, C. Cowan, Ed., pp. 1182-1185. European Association for Signal Processing, 1994.

[10] H. G. Hirsch and C. Ehrlicher, "Noise estimation techniques for robust speech recognition," in Proc. IEEE. Int. Conf. Acoustics, Speech, Signal Processing, 1995, vol. 1, pp. 153-156.

[11] Y. Kaneda and J Ohga, "Adaptive microphone-array system for noise reduction," IEEE Trans. Acoust. Speech Signal Processing, vol. ASSP-34, no. 6, pp. 1391-1400, December 1986.

[12] M. Dahl and I. Claesson, "Acoustic noise and echo canceling with microphone array," IEEE Trans. On Vehicular Technology, vol. 48, no. 5, pp. 1518-1526, September 1999.

[13] B. D. Van Veen and K. M. Buckley, "Beamforming: A versatile approach to spatial filtering," IEEE ASSP Magazine, 1988.

[14] N. Westerlund, M. Dahl, and I. Claesson, "Speech enhancement using an adaptive gain equalizer," in Proc. of DSPCS, December 2003.

[15] N. Westerlund, M. Dahl, and I. Claesson, "Real-time implementation of an adaptive gain equalizer for speech enhancement," in Proc. of WSEAS, December 2003.

[16] L. Deng and D O'Shaugnessy, Speech Processing - A Dynamic and Optimization-Oriented Approach, Marcel Dekker, Inc., New York, 2003. 